\title{
Photodynamic therapy of oral leukoplakia and oral lichen planus using methylene blue: A pilot study
}

\author{
Savarimuthu Wilfred Prasanna \\ Department of Medical Physics \\ Anna University, Chennai-600025, India \\ Ekta Ingle \\ Department of Oral Medicine and Radiology \\ Meenakshi Ammal Dental College \\ Maduravoyal, Chennai-600095, India \\ Prakasa Rao Aruna \\ Department of Medical Physics \\ Anna University, Chennai-600025, India \\ Chidambaranathan Pravada \\ Department of Oral Medicine and Radiology \\ Sathyabama University Dental College, Chennai-600119, India \\ Dornadula Koteeswaran \\ Department of Oral Medicine and Radiology \\ Meenakshi Ammal Dental College \\ Maduravoyal, Chennai-600095, India \\ S. Ganesan \\ Department of Medical Physics \\ Anna University, Chennai-600025, India \\ sganesan@annauniv.edu
}

Received 2 January 2014

Accepted 20 April 2014

Published 30 May 2014

Premalignant lesions like oral lichen planus (OLP), oral leukoplakia (OL) has a fair probability of transforming into malignancy and they are perverse toward conventional therapies. Photodynamic therapy (PDT) has been considered as an alternative/complimentary therapeutic modality for the management of premalignant lesions. In this study, methylene blue-mediated photodynamic therapy (MB-PDT) was used as a possible alternative method for the treatment of OLP and OL. A total of 15 OLP lesions and 13 OL lesions were enrolled in the study. The

This is an Open Access article published by World Scientific Publishing Company. It is distributed under the terms of the Creative Commons Attribution 3.0 (CC-BY) License. Further distribution of this work is permitted, provided the original work is properly cited. 


\section{S. W. Prasanna et al.}

patients were irradiated using metal halide lamp filtered at $630 \pm 10 \mathrm{~nm}$, with a light exposure dose of $120 \mathrm{~J} / \mathrm{cm}^{2}$ per sitting. For the OLP lesions, MB-PDT was performed once a week for four weeks and for the OL lesions, MB-PDT was performed twice a week for three weeks. Lesions were evaluated pre- and post- and at follow-up sessions by changes in sign and symptom scores, and size of lesions. We have observed a $53.3 \%$ of complete reduction in the treated OLP lesions and their decrease in size, sign and symptom score after treatment and at follow-up session was statistically significant. We have also observed complete response for one OL lesion of the 13 treated lesions. The result indicates that MB-PDT is an effective modality in management of OLP and OL. Among the two types of premalignancies treated with MB-PDT, OLP lesions responded much better than that of OL.

Keywords: Photodynamic therapy; methylene blue; oral lichen planus; oral leukoplakia.

\section{Introduction}

Oral cancer is regarded as the most common cancer in India, and it accounts for one-third of total oral cancer occurrences in the world. ${ }^{1}$ The primary etiology in the occurrence of oral lesions is strongly connected with the use of tobacco. The risk factor increases up to 38 times for heavy smokers when compared with nonsmoker. ${ }^{2,3}$ Besides tobacco-related oral cancers, vitamin deficiency, consumption of alcohol and betel nut may also be the possible reasons for the hike in cancer etiology. ${ }^{4}$ In spite of employing various therapeutic advancements such as radiotherapy, chemotherapy or improvement in surgical excision, the survival rate of oral cancers shows only marginal improvement in the past few decades. ${ }^{5,6}$ The limitation in achieving appreciable therapeutic efficacy is overcome by early detection. ${ }^{7}$ Besides early diagnosis, there is a need to improve the current therapeutic modalities of oral lesions at their premalignant level itself. It is worth to note that many of the precancerous lesions such as oral leukoplakia (OL) and oral lichen planus (OLP) are not having definitive treatment protocols. ${ }^{7,8}$

OL is one of the most common precancerous lesions, which appears like an adherent white patch of keratosis. OL will primarily affect oral mucous membrane or tongue. ${ }^{9,10}$ The occurrence of OL is usually habit related and linked with prolonged use of tobacco either smoked or chewed. ${ }^{11}$ Depending on the degree of histological abnormality, OL may also transform into malignancy with a risk probability of $0.13 \%$ to $17.5 \% .^{7}$ Although the conventional surgical modalities are considered to remove OL, it is reported that the follow-up in the longterm treatment has shown that surgically treated lesions developed cancer at a higher rate $(13 \%)$ than that of surgically untreated lesions $(4 \%){ }^{7}$
OLP is a chronic inflammatory disease that often affects the oral mucosa and data reported that there is an occurrence of $0.5 \%$ to $2.2 \%$ among the populace. The female population is affected two folds higher than that of the male population with a peak age range of $30-60$ years. ${ }^{8,12}$ The occurrence of OLP is clinically classified into four based on their presentation: reticular, papular, erythematous (atrophic) and erosive (ulcerated, bullous), ${ }^{13,14}$ of which recticular lesions on the posterior buccal mucosa have the most common occurrence. ${ }^{15}$ The potential of malignant transformation of OLP is reported to vary greatly from $0.4 \%$ to more than $5 \%$ over a period of $0.5-20$ years. $^{8}$ As OLP is related to T-cell mediated immunological disease, corticosteroids, cyclosporine, tacrolimus and retinoids are generally recommended, but the therapeutic outcome is not satisfactory. ${ }^{12,16}$

In this context, there is a need for alternative and/or complementary therapeutic modalitiy to improve the therapeutic efficacy. Photodynamic therapy (PDT) has been considered in the treatment of various cancerous and precancerous lesions. ${ }^{17}$ This is because PDT has advantages such as noninvasive, rapid cytotoxicity, visible tumor destruction, ability to localize in the treatment area, excellent cosmetic results and minimum or no side effects attracts both clinicians and patients. ${ }^{18,19} \mathrm{In}$ PDT, light sensitive organic dye, commonly referred as photosensitizer (PS), is photo-activated by the exposure of light. ${ }^{18}$ The photo-exited PS may cause cytotoxic effects either by type I and/or type II mechanism. ${ }^{6,18-21}$ Using PDT, attempts were made to treat OL and OLP in the presence of Hematoporphyrin derivative (HPD), Aminolevulinic acidprotoporphyrin IX (ALA-PpIX) and Methylene blue. ${ }^{6,8,22}$ Although porphyrin derivatives are 
considered clinically in the treatment of various premalignant and malignant diseases, they have poor absorption in the therapeutic window region and the patient has to be kept in a dark room to avoid photo-allergies, in particular skin photo-toxic effects. $^{22,23}$

Methylene Blue (MB) is a photosensitizing compound, known to be efficient singlet oxygen generators. MB is a good biocompatible agent possibly because cyclic tetrapyrrolic derivatives have an inherent similarity to the naturally occurring porphyrins present in living matter. Consequently, they have little or no toxicity in the absence of light. ${ }^{24} \mathrm{MB}$ can be administered orally in high doses without any toxic effect. ${ }^{16}$ It has the ability to accumulate in tumors due to its affinity to electronegative interfaces. ${ }^{24} \mathrm{MB}$ excitation is known to cause damage to several biomolecules including nucleic acids, proteins and lipids. This damage is believed to be triggered by both type I and type II processes. Data reported by Aghahosseini et al., ${ }^{8,16}$ reveal that there is a necessity to optimize both optical and PS doses for better therapeutic efficacies.

In the present study, OLP and OL were treated with MB-PDT with multiple fractionations and the post-PDT clinical response was evaluated in terms of size of the lesion, sign and symptom score. It is observed that OLP has responded better than OL due to MB-PDT.

\section{Materials and Methods}

\subsection{Patient selection}

The patients selected for this study were from the group of patients, referring to Meenakshi Ammal Dental College and Hospital, Maduravoyal, Chennai, Tamil Nadu, India, seeking medical treatment for OL and OLP lesions. Patients with habit-related lesions (OL) were motivated and counselled to discontinue the habit and were called after one month for re-examination. In the case of OL, patients with moderate and mild lesions were only considered. Patients with OLP lesions which do not respond to conventional medication and therapies (corticosteroids and cyclosporine) were also included in this study after discontinuing the previous medications for at least a month. Patients under the age of 20, with systemic diseases such as diabetics, hypertension, hepatic or renal diseases, patients of any kind of medication for other illness and patients with any kind of allergy were excluded from the study. All the lesions were diagnosed clinically and confirmed histopathologically. A detailed history of the signs, symptoms and duration of the lesions, past medical and dental history were evaluated by oral medicine specialists. The proposal of this MB-PDT study was approved by Meenakshi Academy of Higher Education and research ethics committee and the study has been conducted in full accordance with ethical principles, including the World Medical Association Declaration of Helsinki. The patients were well informed about therapy, and written consent was obtained prior to the study.

\subsection{Light source}

The light source used in this study is equipped with $400 \mathrm{~W}$ metal halide lamp (Crimescope MCS-400, SPEX, Edison 08820, NJ, USA). The white light produced by the lamp was passed through a liquid waveguide and filtered using a $630 \pm 10 \mathrm{~nm}$ bandpass filter. The power of the light at the output end of the liquid waveguide is $100 \mathrm{~mW}$.

\subsection{PDT protocol}

Commercially available MB was used as a PS. Patients were asked to gargle $5 \% \mathrm{MB}$ solution for $5 \mathrm{~min}$ and the lesion was subsequently exposed to red light at $630 \pm 10 \mathrm{~nm}$. The patients were given a light dose of $120 \mathrm{~J} / \mathrm{cm}^{2}$ per fractionation and the exposure was given in such a way that an additional marginal zone of $0.5 \mathrm{~cm}^{2}$ also gets illuminated. Large lesions were illuminated with multiple spots of $1 \mathrm{~cm}^{2}$ and for each irradiation the aforementioned procedure is repeated. PDT was performed weekly twice for three weeks for OL and weekly once for four weeks for OLP lesions. No adverse effects like pain, nausea, discoloring of urine or irritation were observed during or after the MB-PDT for both OL and OLP treatment and hence local anesthesia or pain killers were not given to the patients during or post-PDT.

\subsection{Clinical evaluation}

Clinical evaluations were performed by measuring the lesion (OLP and OL) with a scaled tongue blade and digital photographs prior to and following the PDT. In the case of OLP, the response rate of PDT 


\section{S. W. Prasanna et al.}

was accessed clinically by three different measures, namely, the reduction in sign and symptom scores and the amount of reduction in the size of the lesions. The reduction in sign scores was assessed as suggested by Aghahosseini et al. ${ }^{8}$ The scores were given as follows: score 5 (white striae with erosive area $>1 \mathrm{~cm}^{2}$ ), score 4 (white striae with erosive area $<1 \mathrm{~cm}^{2}$ ), score 3 (white striae with atrophic area $>1 \mathrm{~cm}^{2}$ ), score 2 (white striae with atrophic area $<1 \mathrm{~cm}^{2}$ ), score 1 (mild white striae only), score 0 (no lesions, normal mucosa).

The symptom score of the lesion is from 0 to 3 on visual analogue scale, which is assessed as reported by Gonzáles-Moles and Scully. ${ }^{25}$ The scores under different conditions of symptoms were given as: $3=$ severe symptoms, $2=$ moderate symptoms, $1=$ mild symptoms and $0=$ absence of symptoms.

The therapeutic response of MB-PDT of OL was evaluated after completing six fractionations of PDT. The response is assessed as per the report of Chen et al. ${ }^{6}$ as described as: CR - lack of detectable lesion confirmed by clinical evaluation; PR reduction of lesion by at least $20 \%$ in diameter; $\mathrm{NR}$ - reduction of lesion by less than $20 \%$ in diameter.

All the above lesion responses were evaluated prior to and 1 week following the completion of the

Table 1. Distribution details of the study population for OLP and OL Patients.

\begin{tabular}{|c|c|c|c|}
\hline $\begin{array}{l}\text { Patient } \\
\text { details }\end{array}$ & $\begin{array}{c}\text { Oral lichen } \\
\text { planus }(n=6)\end{array}$ & $\begin{array}{l}\text { Oral leukoplakia } \\
\qquad(n=10)\end{array}$ & $\begin{array}{c}\text { Total } \\
(n=16)\end{array}$ \\
\hline \multicolumn{4}{|l|}{ Age } \\
\hline 20-30 & 3 & 4 & 7 \\
\hline $31-40$ & 0 & 0 & 0 \\
\hline $41-50$ & 2 & 4 & 6 \\
\hline $51-60$ & 0 & 0 & 0 \\
\hline $61-70$ & 0 & 2 & 2 \\
\hline $71-80$ & 1 & 0 & 1 \\
\hline \multicolumn{4}{|l|}{ Gender } \\
\hline Male & 4 & 8 & 12 \\
\hline Female & 2 & 2 & 04 \\
\hline & & & $\begin{array}{l}\text { Total } \\
\text { no of } \\
\quad \text { lesions } \\
\quad(n=28)\end{array}$ \\
\hline \multicolumn{4}{|l|}{ Location } \\
\hline $\begin{array}{l}\text { Buccal } \\
\text { Mucosa }\end{array}$ & 13 & 12 & 25 \\
\hline Tongue & 01 & 01 & 02 \\
\hline Palate & 01 & - & 01 \\
\hline
\end{tabular}

MB-PDT as post-PDT response and 12 week (3 months) as follow up.

\subsection{Statistical analysis}

All the acquired data (sign, symptom scores and reduction in lesion size) of the patients of both OLP and OL (pre- and post-treatment) were statistically analyzed using SPSS software (SPSS 19.0) for clinical significance.

\subsection{Study population}

A total number of 16 patients were considered for this study after careful consideration of the selection procedure. The study population includes 10 adult patients with 13 OL lesions and 6 adult patients with 15 OLP lesions with the duration of the symptoms ranging from 3 months to 1 year. The demographic data and locations of the lesions in each study group are listed in Table 1.

\section{Results}

\section{1. $M B-P D T$ of $O L P$}

For all the selected 15 OLP lesions, MB-PDT was performed for 4 weeks at the rate of one sitting per week by orally allowing MB to adsorb on the surface of the lesion followed by irradiation of red light. The response of the OLP lesions at pre-, post-PDT and the follow up were characterized by measuring the change in the lesion size and marking of sign and symptom scores. The OLP lesion size was measured using a scaled tongue blade. Figure 1 shows the

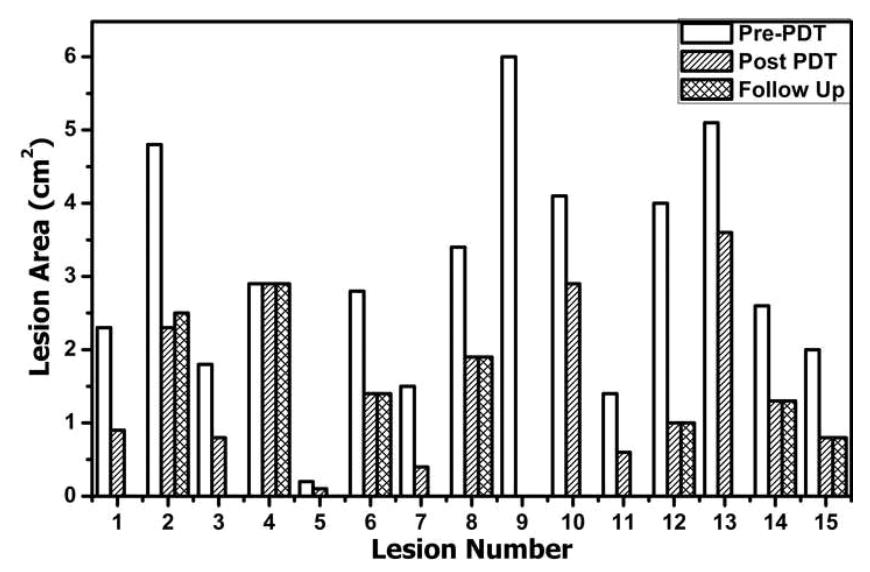

Fig. 1. Size of the OLP lesion at pre-, post-PDT and follow-up conditions. 
$M B-P D T$ for the management of $O L P$ and $O L$

Table 2. Sign score of the OLP lesions at the pre-, post-treated and follow-up conditions.

\begin{tabular}{|c|c|c|c|c|c|c|c|c|c|c|c|c|}
\hline \multirow[b]{3}{*}{ Sign score (pre-PDT) } & \multicolumn{12}{|c|}{ Sign score Post MB-PDT } \\
\hline & \multicolumn{2}{|c|}{ Score 5} & \multicolumn{2}{|c|}{ Score 4} & \multicolumn{2}{|c|}{ Score 3} & \multicolumn{2}{|c|}{ Score 2} & \multicolumn{2}{|c|}{ Score 1} & \multicolumn{2}{|c|}{ Score 0} \\
\hline & $\begin{array}{l}\text { Post- } \\
\text { PDT }\end{array}$ & $\begin{array}{c}\text { Follow- } \\
\text { up }\end{array}$ & $\begin{array}{l}\text { Post- } \\
\text { PDT }\end{array}$ & $\begin{array}{c}\text { Follow- } \\
\text { up }\end{array}$ & $\begin{array}{l}\text { Post- } \\
\text { PDT }\end{array}$ & $\begin{array}{c}\text { Follow- } \\
\text { up }\end{array}$ & $\begin{array}{l}\text { Post- } \\
\text { PDT }\end{array}$ & $\begin{array}{c}\text { Follow- } \\
\text { up }\end{array}$ & $\begin{array}{l}\text { Post- } \\
\text { PDT }\end{array}$ & $\begin{array}{l}\text { Follow- } \\
\text { up }\end{array}$ & $\begin{array}{l}\text { Post- } \\
\text { PDT }\end{array}$ & $\begin{array}{c}\text { Follow- } \\
\text { up }\end{array}$ \\
\hline Score $5(n=1)$ & - & - & - & - & - & - & - & - & - & - & 1 & 1 \\
\hline Score $4(n=2)$ & - & - & - & - & - & - & 2 & - & - & - & - & 2 \\
\hline Score $3(n=11)$ & - & - & - & - & 2 & 2 & 5 & 4 & 4 & 1 & - & 4 \\
\hline Score $2(n=1)$ & - & - & - & - & - & - & - & - & 1 & - & - & 1 \\
\hline
\end{tabular}

histogram of the size of the lesions under pre-, postPDT and follow-up conditions. On the post PDT response, it is observed that there is a considerable reduction in the size for 14 lesions and no reduction in size is observed in one lesion (i.e., $93.3 \%$ lesions exhibit changes and $6.3 \%$ do not exhibit any changes). Further, during the 12 weeks follow-up period it is observed that, 8 lesions have shown complete reduction in size, which is $53.3 \%$ of complete response (CR) in terms of lesion size.

This variation in the lesion size due to PDT is also reflected in the sign score evaluation. The sign score of the OLP lesions under pre-, post-PDT and follow-up conditions were shown in Table 2. It is observed during the post-PDT condition that the lesion with sign score 5 is improved to sign score 0 and two lesions with sign score 4 have improved to score 2. However, during the follow up condition, the lesion change from sign score 5 to score 0 has retained the same score and of the two lesions changed from sign score 4 to score 2 , both of them have improved to score 0 .

Similarly, during the post-PDT, of 11 lesions with sign score 3,5 have improved sign score 2 and 4 have improved to score 1 . However, the same during the follow-up, one lesion which changed from sign score 3 to 2 and three lesions which changed from sign score 3 to 1 have improved to sign score 0 . Further, 2 patients with sign score 3 does not show any change in the sign score during the post-PDT or follow-up conditions. It is also observed that, one lesion with sign score of 2 on pre-PDT has also improved to score 1 during post-PDT and score 0 during the follow-up. It is further observed that there is a negative sign scoring for 13 lesions $(86.7 \%)$ of the 15 treated lesions.

The sign score during pre-, post-PDT and followup was also evaluated in terms of changes in the

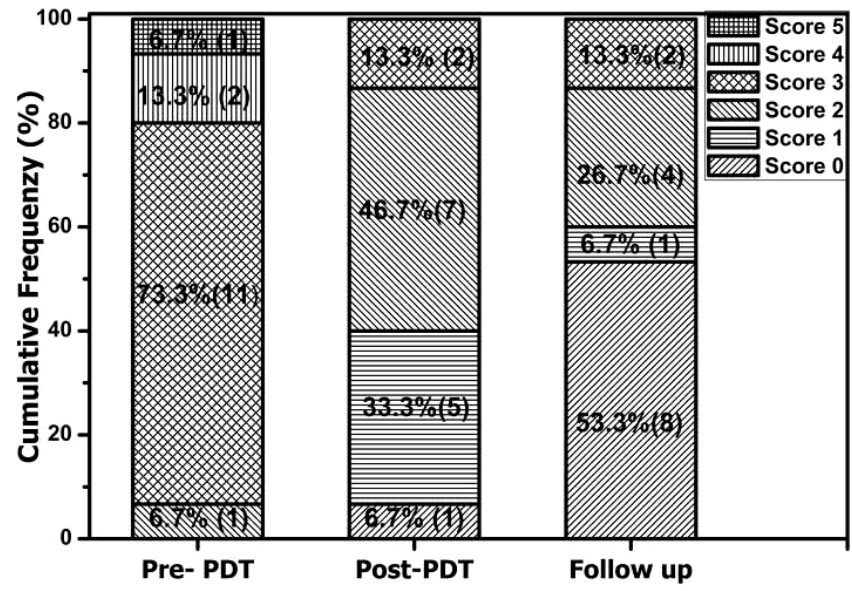

Fig. 2. Frequency of sign score for OLP lesions on pre-, postPDT and follow-up conditions (number of lesions).

cumulative frequency is given in Fig. 2. It is interesting to note that $20 \%$ of erosive lesions with sign score 5 and 4 prior to MB-PDT, were completely absent during post-PDT. It is also observed that the $73.3 \%$ of pre-treated lesions has been reduced to $13.3 \%$ on post-PDT. Further it is observed that the cumulative frequency for scores 1 and 0 were $33.3 \%$ and $6.7 \%$. However, in the follow-up it is observed that, $46.7 \%$ of signs score 2 of post-PDT has reduced to $26.7 \%$ and more significantly, $53.3 \%$ of the treated lesion have shown CR with sign score 0 .

The symptom score was analyzed using visual analogue scale under both pre-, post-PDT and follow-up conditions and they are given in Table 3. It is observed that out of eight lesions with the symptom score 3 , four of them have improved to symptom score 0 and the remaining four lesions have improved to symptom score 1 during postPDT, of the four lesion improved to score 1, two of them have improved to score 0 on follow-up. Similarly of seven lesions with symptom score 2 , six 
Table 3. Symptom score for OLP lesions at the pre-, post-treated and follow-up conditions.

\begin{tabular}{|c|c|c|c|c|c|c|c|c|}
\hline \multirow[b]{3}{*}{ Symptom score (Pre-PDT) } & \multicolumn{8}{|c|}{ Symptom score (Post-PDT) } \\
\hline & \multicolumn{2}{|c|}{ Score 3} & \multicolumn{2}{|c|}{ Score 2} & \multicolumn{2}{|c|}{ Score 1} & \multicolumn{2}{|c|}{ Score 0} \\
\hline & Post-PDT & Follow-up & Post-PDT & Follow-up & Post-PDT & Follow-up & Post-PDT & Follow-up \\
\hline Score $3(n=8)$ & - & - & - & - & 4 & 2 & 4 & 6 \\
\hline Score $2(n=7)$ & - & - & 1 & 1 & - & - & 6 & 6 \\
\hline
\end{tabular}

lesions have improved to symptom score 0 and one lesion with symptom score 2 does not show any change in the symptom score on both post-PDT and follow-up conditions.

The typical photographs that represent the improvement of OLP lesions due to MB-PDT are shown in Fig. 3. The Figs. 3(A1), 3(B1) and 3(C1) are the pre-PDT lesions and their corresponding post-PDT conditions are given in Figs. 3(A2), 3(B2) and $3(\mathrm{C} 2)$ and follow-up are given in Figs. 3 (A3), 3(B3) and 3(C3), respectively. Figure 3(A1) represents the erosive OLP lesion at the palate of the oral cavity prior to the treatment and it completely disappears after MB-PDT [Figs. 3(A2) and $3(\mathrm{~A} 3)$ ]. It is observed that, the treatment site appears smooth with normal oral mucous membrane on both post-PDT and follow-up. In few other cases, the lesion progressed from erosive to atrophic lesion. Figures 3(B1) and 3(B2) shows both pre- and post-treated erosive OLP on buccal mucosa. It is observed that there is a considerable improvement in the severity of the lesion. It is also observed that the pre-treated erosive lesion has changed to mild atrophic lesion. Further on the follow-up condition it is observed that the lesion has responded completely [Fig. 3(B3)] On comparing the pre- and post-treated photographs shown in Figs. $3(\mathrm{C} 1)$ and $3(\mathrm{C} 2)$, it is observed that there is an appreciable reduction in the size of the lesion due to MBPDT, however from Fig. 3(C3) it is observed that
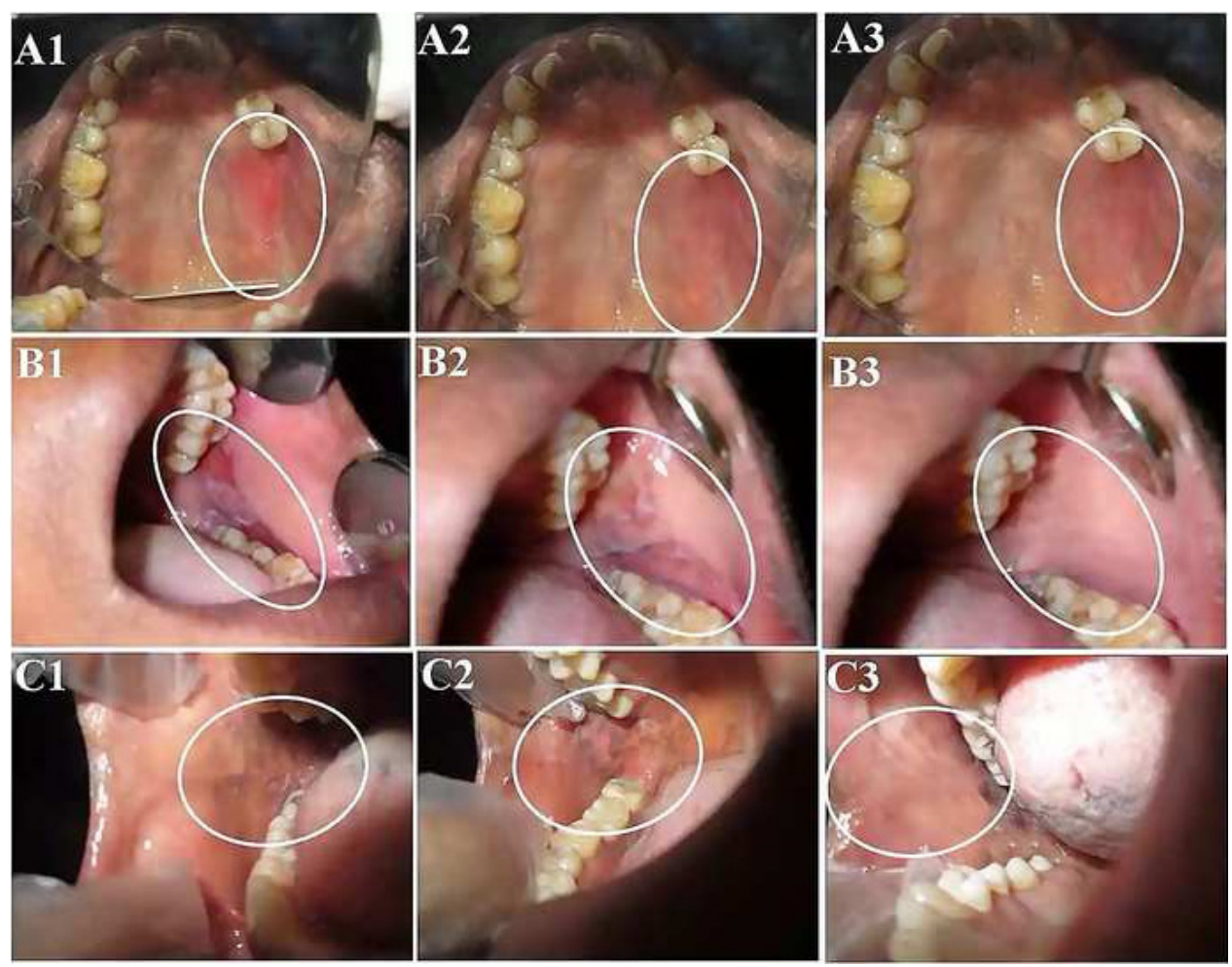

Fig. 3. Clinical photographs of OLP lesion (A1, B1, C1) before the treatment, (A2, B2, C2) and one week after the treatment and (A3, B3, C3) during follow-up, respectively. 
Table 4. Mean of sign, symptom scores and size reduction on pre-, post-PDT and follow-up of the OLP lesions.

\begin{tabular}{lccccc}
\hline & $\begin{array}{c}\text { pre-PDT } \\
\text { Mean } \pm \text { SD }\end{array}$ & $\begin{array}{c}\text { post-PDT } \\
\text { Mean } \pm \text { SD }\end{array}$ & $\begin{array}{c}\text { Follow-up } \\
\text { Mean } \pm \text { SD }\end{array}$ & $\begin{array}{c}\text { Mean } \\
\text { Reduction } \pm \text { SD }\end{array}$ & $\begin{array}{c}\text { Wilcoxon Signed } \\
\text { Rank Test } p \text { value }\end{array}$ \\
\hline Size Reduction & $3.0 \pm 1.6$ & $1.4 \pm 1.1$ & $0.8 \pm 1.0$ & $2.2 \pm 1.7$ & 0.001 \\
Sign Score & $3.2 \pm 0.7$ & $1.7 \pm 0.8$ & $1.0 \pm 1.2$ & $2.2 \pm 1.5$ & 0.001 \\
Symptom Score & $2.5 \pm 0.5$ & $0.5 \pm 0.7$ & $0.4 \pm 0.7$ & $2.1 \pm 0.8$ & 0.001 \\
\hline
\end{tabular}

the lesions have completely reduced in the follow-up condition.

The mean size of the OLP lesions and their sign and symptom scores for pre-, post-PDT and follow-up of the OLP lesions and their mean reduction are given in Table 4. It is observed that, the average value of the size of lesions reduced from a pre-PDT value of $3.0 \pm 1.6$ to a post-PDT value of $1.4 \pm 1.1$ and to $0.8 \pm 1.0$ on the follow-up, the mean reduction of the size of the lesion is $2.2 \pm 1.7$, which indicates $73.3 \%$ of regression in the size of the lesion. This reduction of the size of the lesion mediates significant improvement in mean symptom score from the pre-PDT value of $2.5 \pm 0.5$ to a postPDT value of $0.5 \pm 0.7$ and to $0.4 \pm 0.7$ on the follow-up with a mean reduction symptom score of $2.1 \pm 0.8$. The mean sign score of $3.2 \pm 0.7$ for prePDT reduced into $1.7 \pm 0.8$ at post-PDT and to $1.0 \pm 1.2$ with a mean reduction of $2.2 \pm 1.5$. All the above values are statistically significant with a $p$ value of 0.001 .

\section{2. $\quad M B-P D T$ of $O L$}

The OL lesions were given MB-PDT for three weeks at the rate of two fractionations per week. The therapeutic response was monitored by measuring the reduction in the size of the lesion prior to the treatment and one week after the completion of MB-PDT and after three months as follow-up. The size of the lesions under both pre-, post-PDT and follow-up conditions is shown in Fig. 4.

It is observed that there is an appreciable reduction in the size of the lesions due to PDT. During post-PDT condition, it is observed that out of the 13 OL lesions treated with MB-PDT, only one lesion exhibit a CR, seven lesions exhibit a partial response $(\mathrm{PR})$ and the remaining five lesions have not responded (NR) for PDT. In spite, few of the partially responded lesions have shown similar size in the follow-up condition, it is also observed that four lesions have started reiterating during the follow-up. However, one CR lesion has not recurred.

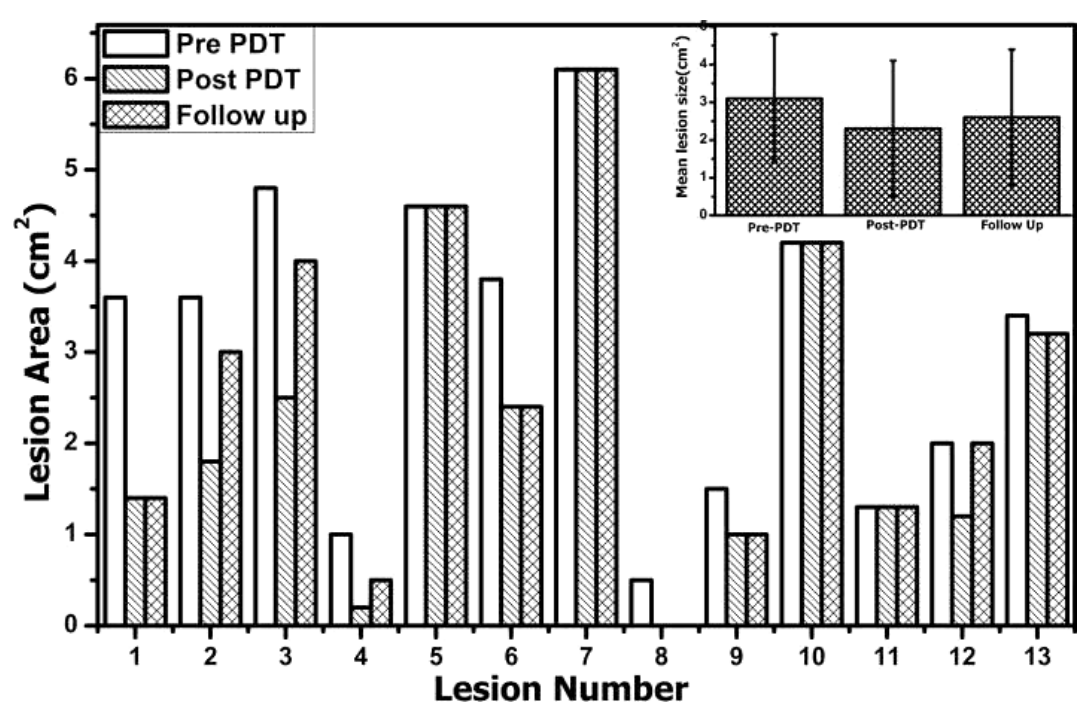

Fig. 4. Size OL lesion at pre-, post-PDT and follow-up conditions. 

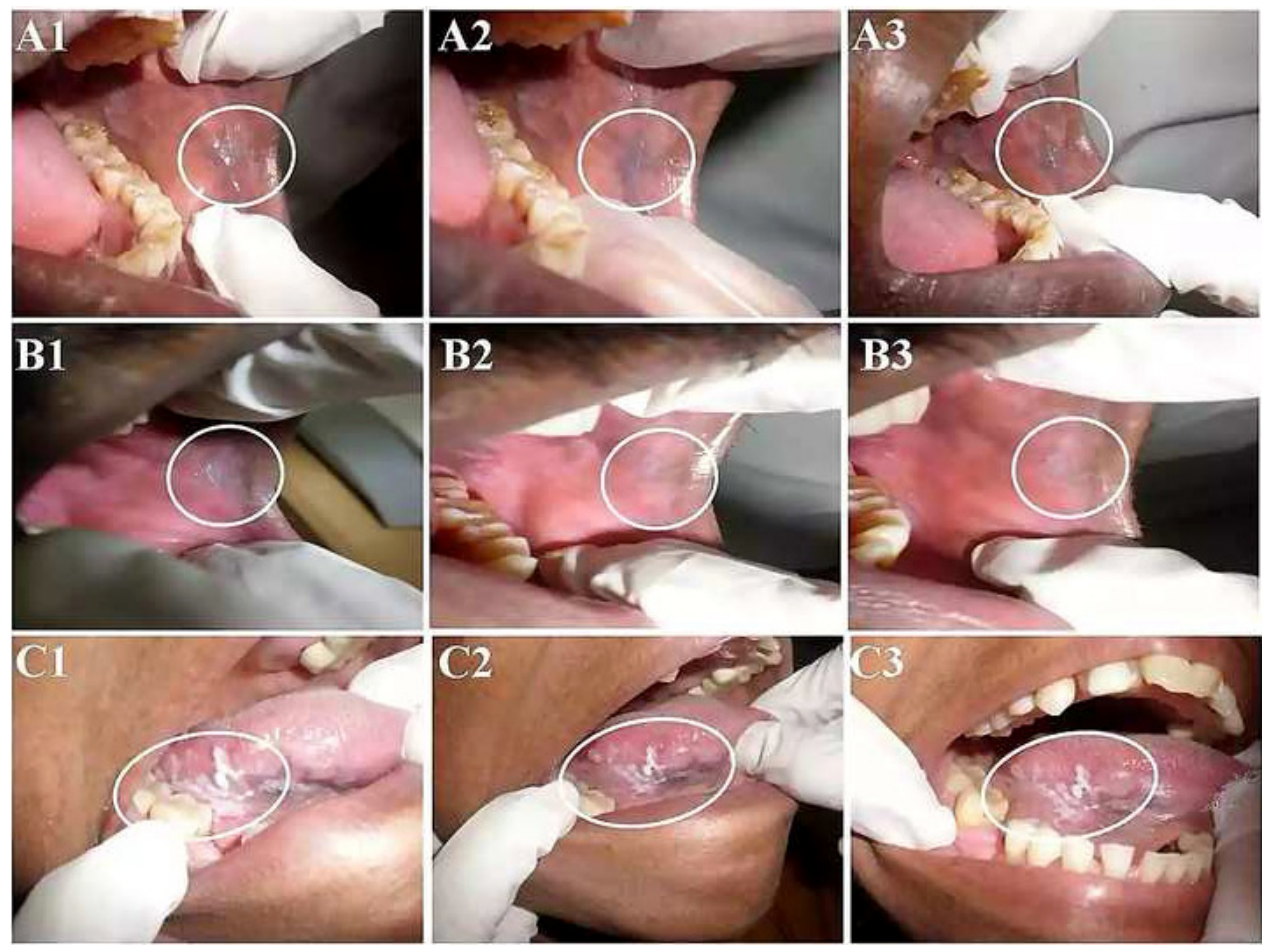

Fig. 5. Clinical photographs of OLP lesion (A1, B1, C1) before the treatment, (A2, B2, C2) and one week after the treatment and (A3, B3, C3) during follow-up, respectively.

This result is also reflected in the mean size of OL lesions during pre-, post-PDT and follow-up conditions of OL lesions, which is given in the inset of Fig. 4. The mean size for 13 OL lesions in the prePDT condition is $3.1 \pm 1.7$ and post-PDT is $2.3 \pm$ 1.8 with a mean reduction of $0.8 \pm 0.8$, However, on the follow-up the mean size of the lesion is $2.6 \pm 1.8$, with a mean reduction of $0.5 \pm 0.6$ and the $p$ value for the per-PDT and follow-up condition is found to be 0.011 , indicating statistical significance.

The typical photographs of the lesions under pre-, post-PDT and follow-up conditions are shown in Fig. 5. Figures 5(A1), 5(B1) and 5(C1) show the OL lesion in pre-PDT conditions and Figs. 5(B1), 5(B2) and 5(B3) show the lesion in post-PDT conditions and Figs. 5(C1), 5(C2) and 5(C3) show lesion in follow-up conditions, respectively. The regression of the lesion can be observed comparing Figs. 5(A1) and 5(A2), where the lesion has lost its color and had disappeared completely on the postPDT itself and no recurrence is observed in the follow-up also as shown in the Fig. 5(A3). Since the above lesions have disappeared completely on visual examination, it is regarded as CR. On comparing Figs. 5(B1) and 5(B2), it is observed that the lesion has faded much to its previous white color and has almost disappeared. However, in the follow-up [Fig. 5(B3)] it is observed that the lesion has not only retained but also has increased its size to greater probability to recur. On comparing Figs. 5 (C1) and 5(C2), the clinical photographs of the lesion on tongue pre- and post-PDT, respectively; it is observed that inspite of the slight regression in the lesion size, the lesion persists even after the full period of the scheduled MB-PDT treatment. Moreover, the lesion has retained in the follow-up [Fig. 5(C3)] also with similar size to that of the postPDT. Since the lesion had not reduced to the desired $20 \%$ of the reduction in size, the response of the lesion has been termed as NR.

\section{Discussion}

Premalignant lesions OLP and OL have common occurrence among the populace and they have a fair probability of transforming into malignant conditions. ${ }^{26}$ The existing treatment modalities for $\mathrm{OL}$ and OLP have their own limitations. For example, topical corticosteroids which seem to be the most reliable treatment for OLP are associated with severe adverse effects. Oral PUVA therapy is one of 
the modalities widely considered to treat various lesions. However, it suffers from limitations of severe nausea and potential to trigger malignancy at higher doses. ${ }^{8}$ Similarly in the treatment of OL, surgery is preferred. However, due to surgical excision of OL the rate of recurrence is as high as $35 \% .^{9}$ In this context, PDT has been considered as an alternative and/or complementary modality to treat various oral lesions. ${ }^{27}$

In most of the clinical trials on the PDT of oral lesions, ALA-PpIX has been used as a PS. However, the ALA-PpIX which is administered clears from the skin and other regions by $48 \mathrm{~h}$. This necessitates that the patients must be protected from sun exposure and other light to avoid unnecessary skin photoallergy. In addition, ALA-PDT is regarded as a painful therapy and ALA-PDT also suffers from deeper penetration of the oral mucosa. ${ }^{22}$ On the other hand, $\mathrm{MB}$ has been used by the medical community due to its ability to be orally administered and its efficiency to accumulate in tumors. It has additional advantages of being an efficient singlet oxygen generator, good biocompatible nature and has absorption in the therapeutic window region. ${ }^{24}$ Based on these, MB has also been considered as PS especially in the PDT of oral lesions. However, still PDT of OLP and OL is not yet clinically, optimized warranting further studies.

In the present study, attempt was made to treat OLP using MB-PDT by delivering an optical dose of $120 \mathrm{~J} / \mathrm{cm}^{2}$ per sitting and the patients were treated for 4 times with a total optical dose of $480 \mathrm{~J} /$ $\mathrm{cm}^{2}$. We have obtained the mean reduction in the size of the lesions during the follow-up as MB-PDT is $2.2 \pm 1.7 \mathrm{~cm}^{2}$, which corresponds to $73.3 \%$ of reduction in the size of the lesion. In a similar clinical study conducted by Aghahosseini et al., ${ }^{8}$ to treat oral lesions using 5\% MB and delivered an optical dose of $120 \mathrm{~J} / \mathrm{cm}^{2}$ to the target site in a single exposure. They reported that an overall treatment response is $42.8 \%$ of reduction in lesion size. The improvement in the reduction of lesion size in our study may be attributed to the delivery of dose in multiple fractionations. It is also observed that there is a continuous improvement of the lesions from post-PDT to the follow-up. Similar result has also been observed in the study reported by Sadaksharam et al. ${ }^{13}$ where they have also observed a constant improvement during the different followups. Further, it is found that there is a negative sign score for 13 lesions and erosive lesions has shown complete disappearance of the lesion (Table 2). It is also observed that two of the erosive lesions have changed to mild atrophic lesion during the post-PDT condition due to MB-PDT [Figs. 3(B1) and 3(B2)]. Such post-treated conditions were also observed by Aghahosseini et $a l .{ }^{8}$ Since erosive OLP lesions are associated with high risk of malignant transformation, even the change of erosive lesion to atrophic nature during the post-PDT may be considered as highly important. The mean pre-PDT symptom score value of $2.5 \pm 0.5$ has come down to $0.4 \pm 0.7$ during follow-up, with a reduction mean of $2.1 \pm 0.8$. This means $93.3 \%$ lesions showed negative symptom scoring, indicating that MB-PDT has a potential to provide excellent symptomatic relief in terms of reducing the pain of OLP patients due to MB-PDT. From the results of the present study, it is observed that the MB-PDT of OLP has a significant beneficial effect in the control of the main signs and symptoms of OLP and has potential in the management of OLP. Since OLP is notorious for relapse we did plan for a six month follow-up for which few patients did not report. However, we did not notice any recurrence for the patients who have reported.

Although data reported on the use of ALA-PpIX on the PDT of OL, to our knowledge the PDT of OL using MB is not yet reported. In this context, attempts were also made to treat 13 OL lesions using MB-PDT with a light dose of $120 \mathrm{~J} / \mathrm{cm}^{2}$ per sitting and the patients were treated twice a week for six sittings. Further, the report of Chen et al. ${ }^{6}$ has concluded that OL lesions respond better to twice a week therapy than once in a week, in this regard in the present work we have performed MB-PDT twice a week for OL. In the present study after the MB-PDT of OL, we have observed CR for only one lesion. In other words, it has $7.69 \%$ of CR in the 13 treated lesions. These results with in contradiction with other similar studies like Kubler et al. ${ }^{22}$ where of the 12 treated lesions applied with 20\% ALA cream 5 lesions have responded completely, which is $41.6 \%$ of CR. In other instance, Sierón et al. ${ }^{28}$ have obtained CR for 14 of the 17 treated lesions with 10\% ALA cream, which is $82.3 \%$ of CR. This difference in the results may be attributed to the difference in light source, PS and mode of application of PS. In both the studies of Kubler et al.,$^{22}$ and Sierón et al.,$^{28}$ they have applied the PS in the form of cream onto the surface of the lesion, which has a better adhesive nature to the lesion and restrain to saliva secretion. ${ }^{22}$ 


\section{S. W. Prasanna et al.}

Although the present study shows that there is a considerable amount of reduction in the size of lesion from a pre-PDT value of $3.1 \pm 1.7$ to a postPDT value of $2.3 \pm 1.8$, during the follow-up it is observed as $2.6 \pm 1.8$ (Fig. 4 inset). This may be due to the lesions that have started reiterating once the PDT is over and it is also observed that few of the partially responded lesions have shown similar size during the follow-up also. Hence, it may be an indication that the partially responded lesion has greater probability to recur. However, one lesion that has shown $\mathrm{CR}$ has not recurred during the follow-up also, indicating MB-PDT may be effective. Hence, this argument may be concluded with more number of samples and longer follow-ups and usage of PS in cream form. Nevertheless, at present it is evident that MB-PDT of OL may be effective in the management of mild and smaller lesions under the present treatment conditions.

\section{Conclusion}

In conclusion, among two oral lesions OLP and OL treated using MB-PDT the treatment of OLP, due to the delivery of light dose $480 \mathrm{~J} / \mathrm{cm}^{2}$, exhibits significant improvement in terms of reduction in the lesion size, sign and symptom score. However OL, even with increased light dose of $720 \mathrm{~J} / \mathrm{cm}^{2}$ does not respond adequately for MB-PDT. It is suggested that the improvement in the therapeutic efficacy of MB-PDT may further be achieved if PDT is considered in conjunction with conventional therapies. However, this study suffers with a small number of samples and short follow-ups. Hence, to support the above results, large number of samples and long follow-ups are needed.

\section{Acknowledgment}

This work was supported by grant from the Department of Atomic Energy - Board of research in Nuclear Sciences (DAE-BRNS) Project (Ref. No. 2009/34/38)12.

\section{References}

1. S. Sujha, R. Sankaranarayanan, B. Bapat, T. Somanathan, G. Thomas, "Cost-effectiveness of oral cancer screening: Results from a cluster randomized controlled trial in India," Bull. World Health Org. 87, 200-206 (2009).
2. S. Warnakulasuriya, G. Sutherland, C. Scully, "Tobacco, oral cancer, and treatment of dependence," Oral Oncol. 41, 244-260 (2005).

3. A. Jemal, F. Bray, M. Melissa, J. Ferlay, E. Ward, D. Forman. "Global cancer statistics," Ca Cancer J. Clin. 32, 69-90 (2011).

4. R. Singh, S. Singh, P. Chattopadhya, S. Kalpana, S. Vijender, S. K. Kulshrestha, R. S. Tomar, R. Kumar, S. Garima M. Viola, P. Daniel, "Tobacco consumption in relation to causes of death in an urban population of north India," Int. J. Chron. Obstruct. Pulmon. Dis. 2, 177-185 (2007).

5. A. Leunig, C. Betz, R. Baumgartner, G. Grevers, W. Issing. "Initial experience in the treatment of oral leukoplakia with high-dose vitamin A and follow-up 5-aminolevulinic acid induced protoporphyrin IX fluorescence," Eur. Arch. Otorhinolaryngol. 269, 327-331 (2000).

6. H. M. Chen, H. Y. Chuan, T. Tsai, Y. C. Hsu, K. R. Cheng, C. P. Chiang, "Topical 5-aminolevulinic acid-mediated photodynamic therapy for oral verrucous hyperplasia, oral leukoplakia and oral erythroleukoplakia," Photodiagnosis Photodyn. Ther. 4, 44-52 (2007).

7. P. Holmstrup, P. Vedtofte, J. Reibel, K. Stoltze, "Long-term treatment outcome of oral premalignant lesions," Oral Oncol. 42, 461-474 (2006).

8. F. Aghahosseini, F. Arbabi-Kalati, L. A. Fashtami, G. E. Djavid, M. Fateh, J. M. Beitollahi, "Methylene blue-mediated photodynamic therapy: A possible alternative treatment for oral lichen planus," Laser Surg. Med. 38, 33-38 (2006).

9. A. Ribeiro, P. Salles, T. Silva, R. Mesquita. "A review of the nonsurgical treatment of oral leukoplakia," Int. J. Dent. 2010, 1-10 (2010).

10. J. Bánóczy, "Exfoliative cytologic changes in oral leukoplakia," J. Dent. Res. 48, 17-21 (1969).

11. H. Stich, B. Mathew, R. Sankaranarayanan, M. Krishna, "Remission of precancerous lesions in the oral cavity of tobacco chewers and maintenance of the protective effect of beta-carotene or vitamin A," Am. J. Clin. Nutr. 32, 298-305 (1991).

12. M. Sahebjamee, F. Kalati, "Management of oral lichen planus," Arch. Iran Med. 32, 252-256 (2000).

13. J. Sadaksharam, K. P. T. Nayaki, N. P. Selvam, "Treatment of oral lichen planus with methylene blue mediated photodynamic therapy - a clinical study," Photodermatol. Photoimmunol. Photomed. 28, 97-101 (2012).

14. M. Carrozzo, R. J. Thorpe, "Update on oral lichen planus," Expert. Rev. Dermatol. 4, 483-494 (2009).

15. C. Scully, M. Beyli, M. C. Ferreiro et al., "Update on oral lichen planus: Etiopathogenesis and management," Crit. Rev. Oral. Biol. Med. 9, 86-122 (1998). 
16. F. Aghahosseini, F. Kalati, L. Fashtami, M. Fateh, G. Djavid, "Treatment of oral lichen planus with photodynamic therapy mediated methylene blue: A case report," Med. Oral. Patol. Oral. Circ. Buccal. 11, 126-129 (2006).

17. R. Allison, G. Downie, R. Cuenca, "Photosensitizers in clinical PDT," Photodiagnosis Photodyn. Ther. 1, $27-42$ (2004).

18. T. J. Dougherty, C. J. Gomer, B. W. Henderson, G. Jori, D. Kessel, M. Korbelik, J. Moan, Q. Peng, "Photodynamic therapy," J. Natl. Cancer Inst. 90, 889-905 (1998).

19. K. Konopka, T. Goslinski, "Photodynamic therapy in dentistry," J. Dent. Res. 86, 694-704 (2007).

20. Z. Luksiene, "Photodynamic therapy: Mechanism of action and ways to improve the efficiency of treatment," Medicina 39, 1137-1150 (2003).

21. S. Ganesan, V. Masilamani, S. Natarajan. "Photodynamic activity on human erythrocytes," Curr. Sci. 57, 639-643 (1988).

22. A. Kubler, T. Haase, M. Rheinwald, "Treatment of oral leukoplakia by topical application of 5-aminolevulinic acid," lnt. J. Oral Maxillofac. Surg. 27, 466-469 (1998).

23. D. Parmeswaran, S. Madhuri, P. Aruna, P. K. Gupta, S. Ganesan, "In vivo pharmacokinetics of 8-aminolevulinic acid-induced protoporphyrin IX during pre- and post-photodynamic therapy in 7,12-dimethylbenz(a)nthracene-treated skin carcinogenesis in Swiss mice: A comparison by threecompartment model," Photochem. Photobiol. 76, 81-90 (2002).

24. J. P. Tardivo, A. D. Giglioa, C. S. Oliveirab, D. S. Gabriellib, H. C. Junqueirab, D. B. Tadab, D. Severinob, R. F. Turchiellob, M. S. Baptista, "Methylene blue in photodynamic therapy: From basic mechanisms to clinical applications," Photodiagnosis Photodyn. Ther. 2, 175-191 (2005).

25. M. A. González-Moles, C. Scully, "Vesiculo-erosive Oral Mucosal Disease - Management with topical corticosteroids: (2) Protocols, monitoring of effects and adverse reactions, and the future," J. Dent. Res. 84, 302-308 (2005).

26. J. E. Bouquot, P. Suarez, N. Vigneswaran, "Oral precancer and early cancer detection in the dental office - Review of new technologies," J. Imp. Adv. Clin. Dent. 2, 47-63 (2010).

27. T. J. Dougherty, J. E. Kaufman, A. Goldfarb, K. R. Weishaupt, D. Boyle, A. Mittleman, "Photoradiation therapy for the treatment of malignant tumors," Cancer Res. 38, 2628-2635 (1978).

28. A. Sierón, M. Adamek, A. Kawczyk-Krupka, S. Mazur, L. Ilewicz, "Photodynamic therapy (PDT) using topically applied $\delta$-aminolevulinic acid (ALA) for the treatment of oral leukoplakia," J. Oral Pathol. Med. 32, 330-336 (2003). 